Ann. Zootech., I969, 18 (3), 359-36r.

\title{
COMPARAISON ENTRE 3 MÉTHODES DE MESURE DE LA SOLIDITÉ DE LA COQUILLE
}

\author{
P. MONGIN \\ Station de Recherches avicoles, \\ Centre national de Recherches zootechniques, 78 -Jouy-en-Josas \\ Institut national de la Recherche agronomique
}

\section{SOMMAIRE}

Tivis méthodes directes sont utilisées pour évaluer la solidité des coquilles de l'vuf : le poids spécifique de l'œuf, le pourcentage de coquille et l'index de solidité ( $\mathrm{g}$ de coquille/Ioo $\mathrm{cm}^{2} \mathrm{~d}^{\prime} \mathrm{euf}$ ).

Nous avons quantifié à partir des valeurs tirées de ces mesures, leur dépendance vis-à-vis du poids de l'ceuf, lour variabilité, la part de leur variance due à la poule et les corrélations entre elles.

Plusieurs critères de mesure de la solidité de la coquille ont été proposés : le poids spécifique de l'œuf, le pourcentage de coquille et l'index de solidité ( $g$ de coquille pour $100 \mathrm{~cm}^{2}$ d'œuf). Nous avons étudié la corrélation qui existe entre chacun de ces 3 critères et le poids de l'œuf.

Nous avons choisi 20 poules Leghorn blanches et fait les 3 mesures sur io oufs de chaque individu. De plus, nous avons intentionnellement pris une gamme de poids d'œuf très étalée de $43,2 \mathrm{~g}$ à $7 \mathrm{r}, 5 \mathrm{~g}$, afin de tester la dépendance entre chacune des trois méthodes et le poids de l'œuf.

\section{RÉSULTATS ET DISCUSSION}

De l'ensemble des résultats présentés dans les deux tableaux ci-dessus, nous pouvons tirer les conclusions suivantes :

I. Le poids spécifique de l'œuf et son index de coquille sont indépendants du poids de l'œuf, alors que le pourcentage de coquille ne l'est pas. Donc, pour une même poule, si le poids de l'œuf est très variable, la comparaison des solidités de coquille exprimées en p. cent du poids de l'œuf sera difficile à interpréter. C'est le cas, par exemple, d'un traitement qui affecte à la fois le poids de l'ouf et celui de sa coquille. 
Ainsi, à pourcentages de coquille égaux, soit $\$, 5$ des oufs de to et to g ont respectivement des index de 6 et de 7 (Moxar, rg65). Tar contre, si les classes de poicls sont les mèmes, on peut utiliser indifféremment l'index ou le pourcentage de coquille, étant donné la forte corrélation $(+0,916)$ entre les deux.

\section{TABLEAU I}

Moyenne, écart-type de la moyenne, variabilitó de chacun des + caractères mesurés et part de la variance due à la poule

\begin{tabular}{|c|c|c|c|c|}
\hline I.ibellés & Moyenne & Ecart-type & Variabilité & $\begin{array}{l}\text { Part } \\
\text { de la variance } \\
\text { due à la Poule }(\%)\end{array}$ \\
\hline Poids de l'ouf $(\mathrm{g}) \ldots \ldots \ldots$ & 60,5 & 0,30 & 6,87 & 70,6 \\
\hline Poids spécifique ....... & 1,081 & $0,000^{\prime}$ & 0,51 & $5: 4,9$ \\
\hline Pourcentage de cocluilie. & 8,77 & $0,05: 3$ & 8,16 & $6,5,2$ \\
\hline Index de solidité ...... & 7,38 & $0,0^{\prime} i^{\prime}$ & $8, \geq 6$ & 67,0 \\
\hline
\end{tabular}

\section{TABLEAU 2}

Coefficients de corrélatiom entre les quatre variables

Dans la moitié supérieure droite coefficient de corrélation intra-poule et dans la moitié inférieure gauche, coefficient de corrélation entre poules c'est-à-dire sur les moyennes

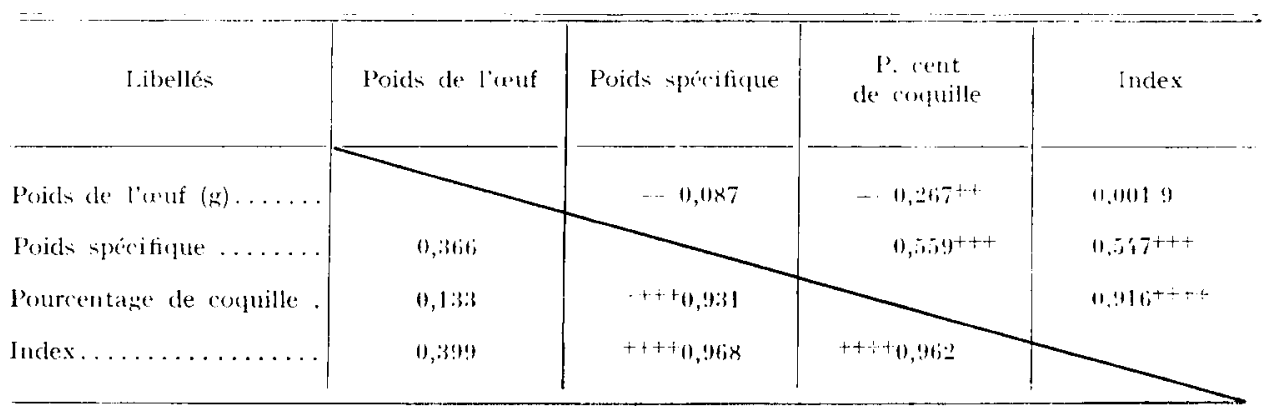

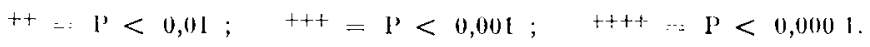

2. Si l'on travaille sur des moyennes par poule, on peut utiliser n'importe quel critère d'appréciation de la solidité. Néanmoins, le poids spécifique présente une très faible variabilité $(0,5 \mathrm{I})$ par rapport au pourcentage de coquille $(8,16)$ et à l'index $(8,26)$. Ceci implique donc unc très grande précision dans la mesure du poids spécifique, en particulier contrôles fréquents de la température et de la concentration des solutions salines (BERNIER, I964).

3. Dans le choix d'une méthode en vue d'une sélection sur la solidité de la coquille, pourcentage et index de coquille sont recommandables en raison de leur variabilité et de la part importante de leur variance qui est liée à l'individu. E'une ou l'autre s'impose dans le cas où l'on sélectionne également sur la qualité de l'albumen: et l'abseńce deśs taches de sang, car ces mesures impliquent 
le cassage de l'œuf. La moyenne du poids spécifique reste néanmoins une bonne méthode, compte tenu des restrictions faites au paragraphe précédent; elle est surtout facile d'emploi.

Quant aux expériences faites en laboratoire, il nous semble que l'index de solidité s'impose.

Rę̧ pour publication en novembre 1969.

\section{SUMMARY}

COMPARATIVE STUDY OF THREE METHODS FOR MEASURING STRENGTH OF EGG-SHELI,

Three methods are used to measure egg shell strength : specific gravity, percentage of shell and weight of shell/10o $\mathrm{cm}^{2}$.

Dependance of egg weight, variability, part of variance due to the hen and correlations between this three methods are measured.

\section{RÉFÉRENCES BIBLIOGRAPHIQUES}

Bernier P. E., I955. How and when to measure shell strength. Eggsaminer, 32 (I), I6-I9.

Mongrn P., ig65. Index de solidité de coquille de l'ouf. Ses significations. Sa précision. Ann. Zootech., 14, 3 I9-325.

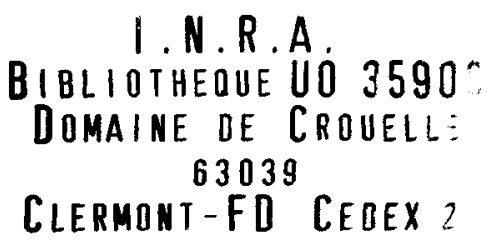

